\title{
Time Series, Multi-Color, and Spectroscopic Observations of a New Possible Double White Dwarf System
}

\author{
Robert F. Wilson ${ }^{* 1}$, Keaton J. Bell ${ }^{1}$, Samuel T. Harrold ${ }^{1}$, A. Gianninas ${ }^{2}$, Mukremin \\ Kilic $^{2}$, Warren R. Brown ${ }^{3}$, J.J. Hermes ${ }^{4}$, M. H. Montgomery ${ }^{1}$, D. E. Winget ${ }^{1}$ \\ ${ }^{1}$ University of Texas at Austin, Department of Astronomy \\ ${ }^{2}$ University of Oklahoma, Department of Physics and Astronomy \\ ${ }^{3}$ Smithsonian Astrophysical Observatory \\ ${ }^{4}$ University of Warwick, Department of Physics
}

\begin{abstract}
We report observations of the eclipsing system SDSS J160036.83+272117.8 (J1600). We obtained low resolution spectra sampled at different orbital phases, time-series photometry of the primary and secondary eclipses, and color photometry in and out of the primary and secondary eclipses. J1600 was originally identified as an extremely low mass (ELM) white dwarf (WD) in a recent ELM survey by its broad Balmer absorption, where the spectral fit indicated atmospheric parameters close to the instability strip [1]. We took time series photometry to assess its variability, and found deep eclipses in the light curve. Binary systems are one of the most important tools to study stellar parameters, and J1600 would be the first opportunity to study ELM WDs in this way. However, preliminary analysis suggests radii inconsistent with ELM models and may point to systematic errors in the methods for identifying ELM WDs. To fully understand this system, we consider solutions of two main sequence (MS) stars, WD+MS systems, and WD+WD systems. If J1600 is a double white dwarf system, this is the seventh discovery of such a system. In these proceedings we describe the observations we have obtained of this eclipsing system.
\end{abstract}

BASH 2015

18 - 20 October, 2015

The University of Texas at Austin, USA

\footnotetext{
* Speaker.
} 


\section{Observations}

We obtained spectra with the $4 \mathrm{~m}$ telescope at Kitt Peak National Observatory, the $6.5 \mathrm{~m}$ Multiple Mirror Telescope, and the $5.1 \mathrm{~m}$ Hale telescope at Palomar Observatory. We have extensive timeseries photometry of the primary and secondary eclipses through a broad BG40 filter and g', r', i', z' photometry in and out of the primary eclipse from the $2.1 \mathrm{~m}$ telescope at McDonald Observatory. We also have well-calibrated Sloan Magnitudes out of eclipse from the Sloan Digital Sky Survey (DR3)

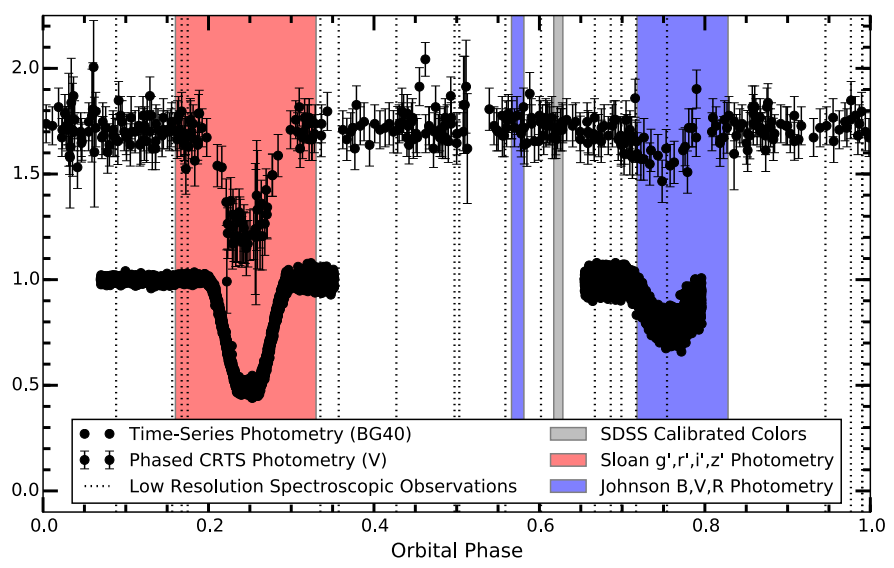

Figure 1: Representation of all observations taken of J1600 plotted in phase with arbitrary offsets. and photometry of this object in the V band from the Catalina Real Time Survey (CRTS). Lastly, we obtained Johnson B, V,R photometry of the secondary eclipse from the $0.6 \mathrm{~m}$ telescope at the Paul and Jane Meyer Observatory. We summarize the distribution of these observations through orbital phase in Figure 1.

\section{Preliminary Analysis}

From the CRTS photometry, we find an orbital period of $1.0037 \mathrm{~d}$. Since the primary and secondary eclipses are $\sim 0.5$ period apart, and the durations are approximately equal, we adopt a model of spherical stars in a highly circular orbit. From the depths and durations of the primary and secondary eclipses, we derive the following constraints, $R_{1}>R_{2}, T_{1}<T_{2}$, and $L_{1} \sim L_{2}$, where we refer to the cooler, larger radius star as the primary star. From the spectra, we measure different radial velocities for different absorption lines, leading to the conclusion that each star contributes strong Balmer absorption, with differing line strengths.

\section{Future Work}

In the primary eclipse all of the contributed flux is from the primary star. Thus the g', ,', i', z' photometry of the primary eclipse gives a spectral energy distribution (SED) of the larger, cooler star which we can compare to stellar models[2]. The difference between this and the photometry out of eclipse gives the SED of the secondary star. Combined with size and mass constraints from the light curve, we are exploring the solution space for stellar parameters that reproduce the measured spectra.

\section{References}

[1] Gianninas, A., et al. "The ELM Survey. VI. Eleven New Double Degenerates." arXiv preprint arXiv:1509.07134 (2015).

[2] Koester, D. "White dwarf spectra and atmosphere models." Memorie della Societa Astronomica Italiana 81 (2010): 921-931. 